\title{
Concepciones de calidad educativa desde la perspectiva docente en la Universidad de Cuenca - Ecuador
}

Conceptions of education quality according teaching perspective in University of Cuenca - Ecuador

\section{Volumen 20, Número 2 \\ Mayo - Agosto \\ pp. 1-22}

\author{
Cristina Cedillo Quizhpe \\ Freddy Cabrera Ortiz \\ Ángel Japón Gualán
}

Citar este documento según modelo APA

Cedillo Quizhpe, Cristina., Cabrera Ortiz, Freddy. y Japón Gualán, Ángel. (2020). Concepciones de calidad educativa desde la perspectiva docente en la Universidad de Cuenca - Ecuador. Revista Actualidades Investigativas en Educación, 20(2), 1-22. Doi. 10.15517/aie.v20i2.41641 


\title{
Concepciones de calidad educativa desde la perspectiva docente en la Universidad de Cuenca - Ecuador \\ Conceptions of education quality according teaching perspective in University of Cuenca - Ecuador
}

\author{
Cristina Cedillo Quizhpe ${ }^{1}$ \\ Freddy Cabrera Ortiz ${ }^{2}$ \\ Ángel Japón Gualán ${ }^{3}$
}

\begin{abstract}
Resumen: El objetivo del presente artículo es describir las concepciones de calidad educativa desde la perspectiva docente en la Universidad de Cuenca, Ecuador. Este estudio descriptivo - correlacional encuestó a 462 docentes de esta universidad, seleccionados a través de un muestreo probabilístico estratificado. Los datos se recolectaron en el 2018, con los bloques cuatro y cinco del cuestionario empleado en el Estudio de la Percepción del Profesorado Universitario sobre las variables asociadas con la calidad de la educación universitaria (EPPU). Para el análisis de datos se empleó estadística descriptiva y pruebas no paramétricas. Los resultados muestran que, respecto a la concepción de la calidad educativa, la mayoría se identifica con la idea de que esta consiste en formar estudiantes capaces de asumir un compromiso social. Así mismo, consideran que la universidad se identifica con la idea de calidad ligada al cumplimiento de los objetivos y la misión institucional. No se encontraron correlaciones de las características del alumnado y la concepción de calidad educativa con las variables sexo, edad y experiencia docente. En cuanto a las características del estudiantado, el profesorado destaca la vocación por los estudios y la actitud del alumnado hacia el aprendizaje lo que genera un efecto sobre la mejora de la calidad educativa según el profesorado. Se concluye que la concepción de calidad del profesorado se inclina por una visión más apegada al desarrollo humano centrada en la formación de sus estudiantes con responsabilidad social.
\end{abstract}

Palabras clave: calidad educativa, concepción de calidad, educación superior, profesorado

Abstract: The aim of this article is to describe the education quality conceptions This descriptive-correlated study applied a survey named, "Cuestionario empleado en el Estudio de la Percepción de Profesorado Universitario sobre variables que afectan a la calidad de la educación universitaria" (EPPU) was given to a sample of 462 professors from the University of Cuenca, in Ecuador. The data was collected on 2018. For data analysis was used descriptive statistic and no parametric test The results show that: the conception of educational quality, most of the professors identify themselves with the idea that quality consists in educating students so that they become capable of assuming a social commitment. Moreover, the academics think that the university, as an institution, is identified with the idea that quality consists in the achievement of the institutional objectives and mission. There were no correlations of the students' characteristics and the conception of educational quality with the variables sex, age and professors' experience. Regarding student's characteristics, professors highlight the vocation for studies and the attitude of students towards learning, which generates an effect on the improvement of educational quality according to professors. The study concludes that quality conceptions according to the university professors show a preference for quality as transformation focused on student training with social responsibility.

Keywords: education quality, quality conceptions, higher education, professors

1 Docente de la Universidad de Cuenca, Ecuador. Dirección electrónica: cristina.cedillo@ucuenca.edu.ec Orcid https://orcid.org/0000-0001-6948-6203

2 Docente de la Universidad de Cuenca, Ecuador. Dirección electrónica: freddy.cabrera@ucuenca.edu.ec Orcid https://orcid.org/0000-0002-7539-0985

3 Docente de la Universidad de Cuenca, Ecuador. Dirección electrónica: ANGEL.JAPON@UCUENCA.EDu.EC Orcid https://orcid.org/0000-0001-6683-5842

Artículo recibido: 29 de octubre, 2019

Enviado a corrección: 23 de marzo, 2020

Aprobado: 27 de abril, 2020

Los contenidos de este artículo están bajo una licencia Creative Commons 


\section{Introducción}

En los años 90, literatura especializada como la de Hughey (1997) describía lo mucho que la educación superior podría aprender del comercio y la industria, sostenía que, si bien no todos los conceptos y estrategias utilizados por el sector comercial pueden ser directamente aplicados al ámbito universitario, valía por lo menos tenerlos en cuenta. A partir de esa década, el discurso de la calidad ha sido una constante dentro de las agendas de las Instituciones de Educación Superior (IES) de los países latinoamericanos. Ecuador no fue la excepción, paulatinamente términos como calidad educativa y aseguramiento de la calidad educativa han permeado cada una de las instancias del contexto universitario. Latinoamérica experimentó, a lo largo de esa década, cambios profundos en el ámbito universitario, marcados por un matiz diferente en la relación Estado - Universidad, que se caracterizó por el rol de la evaluación como dispositivo de regulación, lo que calzó perfectamente en un contexto de reformas neoliberales implantadas en la región y acentuaron los términos calidad, evaluación y acreditación, como un hecho normal dentro de las IES (Leite y Genro, 2012).

En el Ecuador, en el año 2013, se llevó a cabo un nuevo proceso de evaluación y categorización de las universidades a cargo del Consejo de Evaluación, Acreditación y Aseguramiento de la Calidad (CEAACES), sin embargo, los resultados generaron diversas críticas en la comunidad académica ecuatoriana, una de ellas, por ejemplo, cuestionó que el CEAACES no discuta, exponga o defina claramente qué se entiende por calidad en su propuesta (Roig, 2014). Por otro lado, para Villavicencio (2014) cualquier modelo de evaluación debería contemplar la diversidad de instituciones, modalidades pedagógicas, campos disciplinarios y hasta concepciones de calidad diferenciada; el modelo de evaluación aplicado, en ese año, prescindía de esas diferencias.

Aunque la calidad educativa ocupa la atención de las IES y constituye el centro del trabajo de las agencias acreditadoras, no es posible establecer una sola definición de la misma (Crozier, Curvale, Dearlove, Hell y Hénard 2006), de hecho, hay tantas definiciones de calidad en educación superior como categorías de grupos de interés o stakeholders (Brennan, citado en Prisacariu y Shah, 2016) que la definan y perspectivas desde donde la miren. En ese sentido, el presente estudio pretende lograr un acercamiento a la concepción de calidad de los docentes de la Universidad de Cuenca (UC), más aún, cuando la universidad ecuatoriana ha experimentado una serie de cambios durante los diez últimos años; uno de ellos significó, según Ramírez (2013) el cierre definitivo de 14 universidades 
por falta de calidad. En este contexto, el presente estudio encuentra su justificación en la necesidad de indagar sobre las concepciones de calidad del profesorado de la Universidad de Cuenca, Ecuador. Para ello se plantearon tres objetivos: 1) describir las concepciones de calidad educativa universitaria desde la perspectiva del profesorado; 2) identificar las características del alumnado que se consideran determinantes para la calidad según el profesorado; 3) establecer la relación entre características demográficas y las concepciones de calidad del profesorado universitario.

Adicionalmente, es preciso destacar que la UC es una institución de educación superior pública, con una importante incidencia en la región sur del Ecuador, desde donde acoge a estudiantes de las provincias de Azuay, Cañar y El Oro. Finalmente, el artículo está organizado a través de un apartado teórico que presenta una revisión sobre las concepciones de calidad educativa en educación superior, además, de estudios aplicados en distintos contextos. El apartado metodológico detalla los pasos seguidos en la ejecución del estudio; y finalmente, se presentan los resultados y conclusiones a las que se ha llegado con la investigación.

\section{Referente teórico}

En el contexto ecuatoriano las entidades gubernamentales encargadas de evaluar y acreditar a las universidades han acogido la definición de calidad que se describe en el artículo 93 de la Ley Orgánica Reformatoria a Ley Orgánica De Educación Superior (2018), en el mismo se dispone que el "principio de calidad establece la búsqueda continua, autoreflexiva del mejoramiento y construcción colectiva de la cultura de la calidad educativa superior con la participación de todos los estamentos..." (Art. 93)

Las investigaciones en torno a la calidad educativa han sido tantas y tan variadas (Olaskoaga, Marúm y Partida, 2015) intentando aclarar ¿qué es la calidad en la educación superior? Van Kemenade, Pupius y Hardjono (2008) respondieron que probablemente no hay respuesta a esa pregunta o, si la hay, esta no será simple. Para Becher (1999), la calidad es un concepto que conlleva matices políticos que podría representar el poder de varios grupos. En Latinoamérica, el tratamiento de la calidad en ES, ha sido tema de debate y análisis en las diferentes ediciones de las Conferencias Regionales de Educación Superior (CRES). Al respecto, López-Segrera (2012) examinó los distintos documentos generados a partir de las CRES y evidenció la relevancia y evolución que ha tenido el tema en cada uno de los encuentros, partiendo desde la importancia atribuida a la evaluación y la calidad como 
conceptos abstractos y aislados, pasando por enfatizar la garantía de la calidad y la acreditación como un continuo del proceso de evaluación para garantizar la calidad, hasta priorizar la calidad vinculada con valores como: pertinencia, responsabilidad social, equidad y desarrollo sostenible. Según Rovelli (2018), la CRES realizada en Córdova-Argentina en junio del 2018, ratifica la necesidad de abordar la calidad desde un contexto de diversidad, y puntualiza la necesidad de definirla desde todos los actores sociales incluyendo indicadores que contemplen valores como la inclusión, la diversidad y la permanencia.

La definición de calidad constituye un debate actual en el contexto universitario latinoamericano, y es preciso considerar que es un término escurridizo cuyo significado está ligado a los agentes que lo emplean (autoridades, docentes, estudiantes, administrativos, etc.) (Harvey \& Green, 1993). El concepto de calidad se caracteriza porque resulta complejo y confuso de determinar (Olaskoaga et al., 2015). De todas formas, aunque no existe una definición de calidad consensuada, la literatura ofrece algunas perspectivas sobre lo que puede entenderse por calidad educativa.

Según Harvey \& Green (1993) la calidad educativa podría ser entendida como: a) calidad como excepcionalidad, incorpora tres variaciones sobre el concepto de calidad: la primera, una concepción tradicional que entiende a la calidad como distinto y especial, la segunda alude al cumplimiento de estándares que deben ser muy altos y finalmente, una tercera concepción consiste en cumplir un mínimo de estándares; b) calidad como la perfección o la consistencia de los resultados de los procesos, que se orienta hacia la perfección del producto final, sin defectos; c) calidad como adecuación a una finalidad, sugiere que la calidad adquiere significado en relación al producto o servicio, está ligada a las expectativas de los grupos de interés implicados; d) calidad como eficiencia en la utilización de recursos, estrechamente liga a la relación calidad - precio y con el uso eficiente de los recursos, considera la eficiencia y eficacia como valores primordiales; e) calidad como transformación del estudiante, prioriza los cambios cualitativos en lugar de los cuantitativos, considera que la educación es una continua transformación de los participantes no un servicio al cliente.

Cada definición tiene detrás una idea distinta sobre cómo la universidad debe funcionar, optar por alguna de ellas determinará el proceder de un académico en el aula de clase y en la institución (Barrenetxea et al., 2015). En este sentido, Harvey \& Williams (2010) plantean que el análisis de la calidad en la educación superior, requiere que se tome en cuenta dos aspectos: el propósito y el contexto, más aún cuando se trata del concepto en sí 
mismo; para los autores, la calidad es más que la satisfacción de los stakeholders, pues también trae consigo implicaciones políticas.

Las cinco definiciones que hemos descrito de Harvey \& Green (1993), han sido operacionalizadas en una escala que facilita identificar las preferencias de los académicos, administrativos o estudiantes por alguno de los conceptos de calidad, como es el caso del estudio de Barrenetxea et al. (2015) que buscó establecer la relación entre los determinantes socioprofesionales y las concepciones de calidad de académicos españoles; en el mismo se resalta que la edad y el sexo son variables que explican la aceptación de ciertas concepciones de calidad. Entre los hallazgos, describen que las mujeres aceptan definiciones asociadas a la idea de transformación del estudiante a diferencia de los hombres que optan por definiciones ligadas a la consecución de estándares. También, atribuye diferencias en la selección del concepto de calidad según el área de conocimiento de los docentes, formación docente y si ocupan o no un cargo administrativo (Barrenetxea et al., 2015)

Para Cardona, Barrenetxea, Mijangos y Olaskoaga (2009),la concepción de calidad mantiene relación con la satisfacción de las expectativas de los agentes involucrados y con el logro de capacidades en el alumnado que les permita desarrollarse como personas; otra de las conclusiones refiere que la percepción del personal docente sobre la concepción de calidad que tiene la universidad varía notablemente, pues estos consideran que las IES en la que trabajan defiende una idea de calidad ajena a la suya propia. Olaskoaga (2009), por su parte, plantea que al único consenso al que se ha llegado en la comunidad universitaria sobre la calidad, es que es tan difícil de conceptualizarla como medirla, al punto de que todos desean productos y servicios de calidad, aunque no exista unanimidad en su significado. En este sentido, estudios realizados en Argentina, Chile, España y México, resultan interesantes pues, recogen percepciones de docentes sobre el concepto de calidad, calidad de la enseñanza y las variables que están asociadas.

El estudio de Persoglia, López y Zoloa (2009) determinó que los académicos consideran que no existe un concepto de calidad que destaque en la comunidad universitaria para la cual trabajan, todos los conceptos están presentes en similar proporción. Los resultados de Araya, Arias e Ibarra (2009) evidenciaron que los docentes tienen gran diversidad en cuanto al concepto de calidad ya sea entre docentes como la que perciben en las IES. Por otro lado, la investigación de Barrenetxea, Cardona, Mijangos y Olaskoaga (2009) evidenció que el concepto de calidad mantiene una relación con la satisfacción de las 
expectativas de los agentes involucrados y con lograr que los alumnos adquieran capacidades que beneficien su desarrollo, resultados similares a los de Marúm, Cortés, Padilla, Curiel y Robles (2009) que reflejan que la idea de los docentes sobre la calidad, se asocia con la satisfacción de los agentes involucrados (docentes, alumnos y organizaciones, formación de alumnos con compromiso social), pocos de ellos relacionan la calidad en términos de eficiencia).

La calidad educativa también ha evidenciado la realidad en la que se desenvuelven las IES. Plantear la concepción de calidad en educación superior obliga a repensar dos componentes que son inherentes a la realidad universitaria: el desarrollo y el bienestar; elementos que se evidencian al encontrar universidades que optan por trabajar por valores utilitaristas o IES en las que priman concepciones más humanistas para las funciones universitarias. Según Barandiaran (2013), el debate sobre calidad debería considerar también la visión de los agentes internos: por un lado, se puede evidenciar agentes universitarios que atribuyen un valor primordial a la transformación del individuo y el contexto, en ese sentido, definen su concepción de calidad que a la postre impacta en el modelo de desarrollo que se implemente para lograrla. Por otro lado, Boni y Gasper (2011) manifiestan que la visión de universidad apegada a la competitividad y eficiencia es la más generalizada; la visión reduccionista de la IES que prioriza el rol económico que juega la universidad en la formación de individuos se ve reflejada en la medición de la excelencia, prueba de ello son los criterios que manejan los rankings mundiales: número de publicaciones, galardones obtenidos por docentes y alumnos. En fin, el debate teórico y metodológico sobre la calidad de la educación superior es permanente, según AlvaradoLagunas, Luyando-Cuevas y Picazzo-Palencia (2015) tanto por el carácter intangible del producto que se valora, como por la variedad de formas para medirla.

Las investigaciones revisadas, además de indagar sobre la concepción de calidad, también se han concentrado en determinar si las características del alumnado se relacionan con la calidad educativa. Para Marúm et al. (2009) el profesorado tiene una visión clara del tipo de alumnado que requieren los procesos de calidad De acuerdo con Persoglia et al. (2009) el personal docente al valorar las características del alumnado relacionadas con la mejora de la calidad, atribuyen mayor importancia a aspectos actitudinales que a cognitivos formales del estudiantado, en materias como economía y matemáticas. Los resultados de Araya et al. (2009) referidos a la muestra chilena, denotan que el personal docente atribuye 
importancia preponderante a la manera cómo el estudiantado encara su proceso de aprendizaje.

Cardona et al. (2009) analizan la situación del alumnado y cómo ésta se vincula con la calidad de la enseñanza universitaria; para el personal docente participante, uno de los factores mayormente relacionados con la calidad, obedece a la actitud y vocación del alumnado por los estudios. La revisión de Garbanzo (2007) ratifica que conocer el rendimiento académico es un indicador clave de la calidad educativa, no el único, pero sí constituye una dimensión indispensable en el análisis de cómo se concibe la calidad educativa en una IES. Entre los factores asociados al rendimiento académico, llama la atención que la nota de acceso a la universidad, así como, el rendimiento académico previo sean predictores del rendimiento académico de los universitarios; además, la elección de los estudios, según el interés del estudiante, es determinante en el rendimiento académico y en la calidad, es decir, el hecho de si la carrera fue la primera elección del estudiante, si fue por traslado de carrera o por no haber encontrado cupo en otra carrera.

Si bien, no se han encontrado estudios que determinen asociación entre la concepción de calidad y el área de conocimiento, es pertinente anotar lo planteado por Araujo y Trotta (2011) en cuanto a que, cada campo de conocimiento tiene un modo de vida. Quienes ingresan a un campo disciplinario lo hacen también a ambientes culturales distintos que comparten tendencias en torno a los problemas, teorías, metodologías y técnicas de investigación dentro de una disciplina, ello implica conocer el mismo vocabulario, niveles de desarrollo diferentes de estructuración e integración simbólica. El mismo estudio evidencia que, en el caso de las ingenierías, la aplicación de la racionalidad tecnológica para encarar los problemas educativos similares a su actividad profesional, parece estar en la base de la legitimación de la evaluación y la acreditación universitaria. Bajo ese mismo razonamiento podría pensarse que la concepción de calidad a la que se adscribe las áreas técnicas se corresponde con una concepción de calidad más pragmática. Para Neave (2001) citado en Rengifo-Millán (2017) las IES, facultades y programas académicos conformados por varias disciplinas mantienen discusiones teóricas y paradigmáticas que se ven reflejadas en la toma de decisiones y complejizan la organización universitaria. En palabras de Rengifo - Millán (2017) el planteamiento anterior explica por qué los procesos de calidad dependen de las posturas de los distintos grupos disciplinarios y del grado de participación que éstos tengan en planes y actividades de mejoramiento. Finalmente, es necesario tener presente, siguiendo 
a Aguila (2005) que el concepto de calidad en la universidad cambia de contenido en cada época, no es estable ni duradero.

\section{Metodología}

En el presente estudio se empleó un enfoque es de tipo cuantitativo, debido a que esta modalidad recurre a procedimientos y pasos que permiten establecer relaciones del hecho estudiado (McMillan \& Schumacher, 2005), en este caso referidas a las concepciones de calidad en los docentes universitarios. Se corresponde con un diseño no experimental y trasversal; se consideró un alcance correlacional en razón de que este tipo de estudio facilita descubrir y evaluar las relaciones existentes entre las variables que intervienen en el fenómeno (Mateo, 2009). El estudio se desarrolló en el marco del proyecto denominado: La evaluación y acreditación en la Educación Superior: el caso de la Universidad de Cuenca, avalado en el XV Concurso universitario de investigación financiado por la Dirección de Investigación de la Universidad de Cuenca (DIUC).

\subsection{Población y muestra}

El estudio se llevó a cabo en la Universidad de Cuenca, Ecuador con una muestra representativa de 462 docentes, seleccionados a través de un muestreo probabilístico estratificado por facultades, de un tamaño de universo de 1153. El tamaño de la muestra se obtuvo con un margen de error del $3.53 \%$ y un nivel de confianza del $95 \%$. De los participantes, 246 son hombres y 216 mujeres; 173 docentes tienen titularidad y 289 laboran bajo la modalidad de contrato. Los datos fueron recolectados entre los meses de junio, julio y septiembre de 2018.

\subsection{Recolección de datos}

Para la recolección de datos se utilizó el bloque cuatro (12 ítems) y cinco ( 7 ítems) del cuestionario desarrollado por Olaskoaga (2009) y empleado en el Estudio de la Percepción del Profesorado Universitario (EPPU), de ahí que se identifique al instrumento con las iniciales correspondientes al estudio. El bloque cuatro se denomina: Características del alumnado y valora la percepción del profesorado sobre cuestiones relacionadas con el alumnado y la incidencia que el profesorado atribuye a cada uno de los ítems sobre la mejora de la calidad de la educación universitaria. El alfa de Cronbach para el bloque cuatro referido a las características del alumnado fue de 0,87 y para los efectos en la calidad 
educativa de dichas características fue de 0,94 mientras que, el bloque cinco denominado: Concepción de calidad del profesor y de la institución universitaria, que solicita al profesorado valorar el grado en el que se identifica con las siete concepciones de calidad, y en el grado en que creen lo hace la universidad en la que laboran. En los dos bloques, el cuestionario está estructurado mediante una escala Likert valorada desde "muy bajo" =0 hasta "muy alto" =4. Además, el cuestionario incluyó un apartado inicial orientado a recabar información básica de los participantes, junto al área de conocimiento en la que enseña, los años de experiencia docente, etc., información que se correlacionó con los resultados. El rango de tiempo empleado para llenar el cuestionario fue de 10 a 20 minutos.

El alfa de Cronbach para el bloque cinco relativo a las concepciones de los docentes sobre calidad educativa fue de 0.82 y para la concepción de calidad de la universidad según los docentes fue de 0.83 . En cuanto a los aspectos éticos, previo a la recolección de datos se entregó a los participantes los formularios de consentimiento informado en el cual se detalló los principios éticos considerados en el estudio.

\subsection{Procesamiento de datos}

El tratamiento estadístico se realizó con el Software IBM SPSS versión22. Para fines didácticos, se promediaron los valores de la escala 0 a 4 de calidad educativa (media) y de ellos se extrajo la frecuencia relativa porcentual (\%). Para presentar las asociación de la escala de concepción de calidad educativa (ordinal) con las variables sociodemográficas: carreras del área médica y de salud, carreras del área de ciencias sociales o carreras del área técnica (dicotómicas), se empleó el coeficiente de correlación biserial por rangos ( $r_{\text {bRANK }}$ ) que se calcula de manera similar a la correlación por rangos de Spearman (Palmer, Jiménez y Montaño, 2000), por lo que, se representa como 'correl.'. El coeficiente de correlación muestra la relación positiva o negativa entre dos variables a través de un índice que va de -1 a 1 (Martínez, Castellanos y Chacón, 2015); los valores, positivos o negativos, se interpretan de 0 a 0,2 como muy baja correlación, de 0,21 a 0,40 como baja correlación, de 0,41 a 0,6 como correlación media, de 0.61 a 0.8 como alta correlación y 0,81 a 1,0 como muy alta correlación. 


\section{Resultados}

\subsection{Perfil de los encuestados}

De acuerdo al área en la que enseña el personal docente, se encontró que el 26,2 \% lo hace en ciencias médicas y de la salud, es decir, en las carreras de: medicina, imagenología, enfermería, tecnología médica, fisioterapia, fonoaudiología, estimulación temprana, odontología, agronomía y veterinaria; el $24,7 \%$ se ubica en el área técnica que incluye a las carreras: ingeniería ambiental, bioquímica, industrial, civil, comercial, telecomunicaciones, sistemas, marketing, contabilidad y administración, así como también se incluyó a arquitectura.

El 49,1\% incluye a las ciencias sociales en las que se han incluido a: gastronomía, turismo, diseño de interiores, diseño gráfico, artes visuales, danza, psicología clínica, psicología educativa, psicología social, derecho, género, trabajo social, economía, sociología, educación general básica, ciencias de la educación con mención en matemática, cultura física, historia, filosofía, educación inicial, lengua española, comunicación social, cine y al instituto de lenguas. El 72,5\% manifestó haber estudiado en una universidad pública. EI $85,7 \%$ ha alcanzado el título de cuarto nivel o maestría como el grado más alto de estudios. Por otro lado, en cuanto a la filiación política, el $25,5 \%$ manifestó tener una filiación política de izquierda, el $26, .2 \%$ señaló ser de centro y el $8,4 \%$ dijo pertenecer a una tendencia de derecha.

\subsection{Concepciones de la calidad educativa}

En cuanto a la concepción de calidad educativa que dice tener el personal docente de la Universidad de Cuenca (Tabla 1), se encontró que el mayor porcentaje $(80,18 \%)$ afirma que la calidad consiste en formar estudiantes capaces de asumir un compromiso social; otra preferencia de definición fue considerar que la calidad consiste en desarrollar las capacidades del alumnado para avanzar en su propia transformación (79,25\%). Por debajo de estas dos definiciones, se eligieron las opciones que plantean que la calidad consiste en satisfacer las expectativas de todos los involucrados (alumnos, docentes, instituciones, sociedad), la calidad consiste en el cumplimiento de los objetivos y la misión que ha establecido la institución, la calidad consiste en el cumplimiento y la mejora de estándares establecidos o la calidad consiste en satisfacer las expectativas de alumnado y de las organizaciones donde se colocan estudiantes egresados. Por otro lado, el porcentaje más bajo $(65,33 \%)$ representa a aquellos docentes que consideran que la calidad consiste en 
conseguir la eficiencia, es decir, obtener los mejores resultados al menor costo. De este apartado de concepciones de calidad puede destacarse que, para el profesorado es mucho más importante formar estudiantes para asumir compromisos sociales que obtener los mejores resultados con el menor costo posible.

\section{Tabla 1}

Porcentajes y puntuación media del personal docente de la Universidad de Cuenca por cada concepción de calidad, Universidad de Cuenca - Ecuador, 2018

\begin{tabular}{lcc}
\hline Concepciones de calidad & $\%$ & Media \\
\hline $\begin{array}{l}\text { La calidad consiste en el cumplimiento y la mejora de estándares } \\
\text { establecidos }\end{array}$ & 71.08 & 2.84 \\
$\begin{array}{l}\text { La calidad consiste en desarrollar las capacidades del estudiante para } \\
\text { avanzar en su propia transformación }\end{array}$ & 79.25 & 3.17 \\
$\begin{array}{l}\text { La calidad consiste en formar estudiantes capaces de asumir un } \\
\text { compromiso social }\end{array}$ & 80.18 & 3.21 \\
$\begin{array}{l}\text { La calidad consiste en satisfacer las expectativas de los alumnos y de las } \\
\text { organizaciones donde se colocan los egresados }\end{array}$ & 71.08 & 2.84 \\
$\begin{array}{l}\text { La calidad consiste en satisfacer las expectativas de todos los involucrados } \\
\text { (alumnos, docentes, instituciones, sociedad) }\end{array}$ & 77.18 & 3.09 \\
$\begin{array}{l}\text { La calidad consiste en el cumplimiento de los objetivos y la misión que ha } \\
\text { establecido la institución }\end{array}$ & 73.58 & 2.94 \\
$\begin{array}{l}\text { La calidad consiste en conseguir la eficiencia: obtener los mejores } \\
\text { resultados al menor costo }\end{array}$ & 65.33 & 2.61 \\
\hline
\end{tabular}

\% representa la frecuencia relativa porcentual obtenido de dividir la media para cuatro que es el valor más alto esperado.

Fuente: $x x x x x$

En la tabla 2 se presenta porcentaje de identificación que la universidad asume en cuanto a la concepción de la calidad educativa, según el profesorado. El mayor porcentaje de docentes $(72,75 \%)$ señala que la universidad se identifica con la concepción de la calidad que consiste en el cumplimiento de los objetivos y la misión que ha establecido la institución; también se encontró que la calidad consiste en satisfacer las expectativas del alumnado y de las organizaciones donde se colocan a estudiantes egresados $(70,18 \%)$. De forma cercana a este valor $(69,9 \%)$, se encontró que para la universidad la calidad, según el personal docente, es el cumplimiento y la mejora de estándares establecidos. También se advierte aquellos casos, en los cuales el personal docente considera que para la universidad la calidad consiste en formar estudiantes capaces de asumir un compromiso social, satisfacer las expectativas de todos los involucrados (alumnos, docentes, instituciones, sociedad), así como, conseguir la eficiencia obteniendo los mejores resultados al menor costo. Sin embargo, en todos estos casos, el porcentaje es menor al $70 \%$. En efecto, el porcentaje más bajo de todos $(67,43 \%)$ corresponde a la visión de los docentes que consideran que la 
universidad tiene una concepción de calidad orientada a desarrollar las capacidades del estudiantado para avanzar en su propia transformación. Por lo tanto, se concluye que, desde la visión del profesorado, para la institución lo primero es cumplir con los objetivos y misión planificados y luego, el desarrollo de las capacidades del estudiantado para su propia transformación.

Tabla 2

Porcentaje y puntuación media del personal docente sobre el grado de identificación de su universidad por cada concepción de calidad, Universidad de Cuenca-Ecuador, 2018

\begin{tabular}{lcc}
\hline Concepciones de calidad & $\%$ & Media \\
\hline $\begin{array}{l}\text { La calidad consiste en el cumplimiento y la mejora de estándares } \\
\text { establecidos }\end{array}$ & 69,90 & 2,80 \\
$\begin{array}{l}\text { La calidad consiste en desarrollar las capacidades del estudiante para } \\
\text { avanzar en su propia transformación }\end{array}$ & 67,43 & 2,70 \\
$\begin{array}{l}\text { La calidad consiste en formar estudiantes capaces de asumir un } \\
\text { compromiso social }\end{array}$ & 69,35 & 2,77 \\
$\begin{array}{l}\text { La calidad consiste en satisfacer las expectativas de los alumnos y de las } \\
\text { organizaciones donde se colocan los egresados }\end{array}$ & 70,18 & 2,81 \\
$\begin{array}{l}\text { La calidad consiste en satisfacer las expectativas de todos los involucrados } \\
\text { (alumnos, docentes, instituciones, sociedad) }\end{array}$ & 69,30 & 2,77 \\
$\begin{array}{l}\text { La calidad consiste en el cumplimiento de los objetivos y la misión que ha } \\
\text { establecido la institución }\end{array}$ & 72,75 & 2,91 \\
$\begin{array}{l}\text { La calidad consiste en conseguir la eficiencia: obtener los mejores } \\
\text { resultados al menor costo }\end{array}$ & 68,30 & 2,73 \\
\hline
\end{tabular}
Fuente: $x x x x$

La tabla 3 muestra las relaciones existentes entre la concepción de calidad educativa que tiene el docente según el área en la que enseña, mediante el coeficiente de correlación biserial por rangos ( $\mathrm{r}_{\mathrm{bRANK}}$ ) no paramétrico que presentamos como 'correl.'. De este modo, el personal docente que enseña en las carreras médicas y de la salud tiene una correlación negativa muy baja con la concepción de que la calidad consiste en desarrollar las capacidades del estudiante para avanzar en su propia transformación y con la concepción de la calidad como la formación de estudiantes capaces de asumir un compromiso social. Por otro lado, la concepción de calidad concerniente al cumplimiento y la mejora de estándares establecidos, muestra una correlación muy baja con el colectivo de docentes del área de las técnicas; es decir, ser docente de esta área, está asociado con la concepción de estándares. Finalmente, ser docente del área de ciencias sociales de la UC está asociada a la concepción de calidad orientada a la formación de estudiantes capaces de asumir un compromiso social, así como con la concepción de satisfacer las expectativas de todos los involucrados (alumnos, docentes, instituciones, sociedad). 
Tabla 3

Correlación biserial por rangos no paramétrico entre las concepciones de calidad y el perfil del profesorado, Universidad de Cuenca - Ecuador, 2018

Concepciones de calidad

\section{Concepciones de calidad del profesorado}

\begin{tabular}{|c|c|c|c|c|c|c|c|}
\hline -a calidad consiste en el cumplir & &,- 066 & 148 &,- 069 &,- 060 &,- 034 & ,082 \\
\hline mejora de estándares establecidos. & & &, 00 & & 208 & 475 & ,085 \\
\hline La calidad consiste en desarrollar las & Correl. & & 03 & 6 &,- 027 & ,009 & ,017 \\
\hline $\begin{array}{l}\text { capacidades del estudiante para avanzar en } \\
\text { su propia transformación. }\end{array}$ & Sig. bil. & ,022 & ,468 & 160 & ,571 & 856 & ,729 \\
\hline $\begin{array}{l}\text { La calidad consiste en formar estudiantes } \\
\text { capaces de asumir un compromiso social. }\end{array}$ & $\begin{array}{l}\text { Correl. } \\
\text { Sig. bil. }\end{array}$ & $\begin{array}{c}-, 098^{*} \\
, 036\end{array}$ & $\begin{array}{r}-, 055 \\
, 238\end{array}$ & $\begin{array}{l}, 133^{\star *} \\
, 004\end{array}$ & $\begin{array}{l}-, 040 \\
, 403\end{array}$ & $\begin{array}{l}, 023 \\
, 631\end{array}$ & $\begin{array}{l}, 016 \\
, 744\end{array}$ \\
\hline $\begin{array}{l}\text { La calidad consiste en satisfacer las } \\
\text { expectativas de los alumnos y de las }\end{array}$ & Correl. &,- 064 & 046 & 016 &,- 062 & 037 & ,022 \\
\hline $\begin{array}{l}\text { organizaciones donde se colocan los } \\
\text { egresados. }\end{array}$ & Sig. bil. & 173 & 321 & 727 & 197 & 436 & 638 \\
\hline $\begin{array}{l}\text { La calidad consiste en satisfacer las } \\
\text { expectativas de todos los involucrados } \\
\text { (alumnos, docentes, instituciones, sociedad). }\end{array}$ & $\begin{array}{l}\text { Correl. } \\
\text { Sig. bil. }\end{array}$ & $\begin{array}{l}-, 086 \\
, 065\end{array}$ & $\begin{array}{l}-, 026 \\
, 586\end{array}$ & $\begin{array}{l}, 098^{*} \\
, 037\end{array}$ & $\begin{array}{l}, 042 \\
, 377\end{array}$ & $\begin{aligned}-, 027 \\
, 569\end{aligned}$ & $\begin{array}{l}-, 014 \\
, 766\end{array}$ \\
\hline $\begin{array}{l}\text { La calidad consiste en el cumplimiento de los } \\
\text { objetivos y la misión que ha establecido la } \\
\text { institución. }\end{array}$ & $\begin{array}{l}\text { Correl. } \\
\text { Sig. bil. }\end{array}$ & , 030 & $\begin{array}{l}059 \\
, 205\end{array}$ & $\begin{array}{l}-, 077 \\
, 100\end{array}$ & $\begin{array}{l}-, 036 \\
, 449\end{array}$ & $\begin{array}{l}, 018 \\
, 708\end{array}$ & $\begin{array}{l}017 \\
, 727\end{array}$ \\
\hline $\begin{array}{l}\text { La calidad consiste en conseguir la eficiencia: } \\
\text { obtener los mejores resultados al menor costo. }\end{array}$ & $\begin{array}{l}\text { Correl. } \\
\text { Sig. bil. }\end{array}$ & $\begin{array}{l}035 \\
, 456\end{array}$ & $\begin{array}{l}, 036 \\
, 444\end{array}$ & $\begin{array}{l}-, 061 \\
, 190\end{array}$ & $\begin{array}{r}-, 007 \\
, 881\end{array}$ & $\begin{array}{r}-, 021 \\
, 661\end{array}$ & $\begin{array}{l}, 024 \\
, 613\end{array}$ \\
\hline
\end{tabular}

*La correlación es significativa en el nivel 0,01 (2 colas).

**La correlación es significativa en el nivel 0,05 (2 colas).

Fuente: $x x x$

Considerando la concepción de calidad educativa de los propios docentes y la concepción de calidad educativa que atribuyen a la universidad, estas no tienen ninguna correlación con variables como: sexo, edad y experiencia docente. Así mismo, no se advirtió correlación de la concepción de la calidad educativa con las variables como ser profesor titular, haber estudiado en una universidad pública, tener una orientación política definida o haber alcanzado un grado académico universitario.

Adicionalmente, se indagó sobre la percepción del personal docente respecto a las características del alumnado y cómo estas se asocian con la mejora de la calidad educativa de la educación superior. 


\subsection{Percepción del profesorado sobre características de los estudiantes y su efecto sobre la mejora de la calidad educativa}

En cuanto a las características que tiene el alumnado y su efecto sobre la mejora de la calidad educativa (Tabla 4), según el punto de vista del personal docente se advirtió que, por arriba del $50 \%$, el personal docente otorga trascendencia a las características del alumnado relacionadas con: la vocación que tienen por los estudios seleccionados, la actitud hacia la innovación, la participación activa del estudiante en las clases presenciales en el periodo universitario, la actitud hacia el aprendizaje al ingreso en la universidad, así como la participación activa del alumnado en su aprendizaje en el periodo universitario. Por debajo del $50 \%$, el personal docente enfatiza las características del alumnado enfocadas en: la capacidad del alumnado para el diseño de su trayectoria formativa, la preparación para el trabajo en equipo a su ingreso en la universidad, los conocimientos de matemáticas al ingreso, el uso de las tutorías por parte del alumnado, la capacidad para el aprendizaje autónomo a su ingreso en la universidad y, los conocimientos generales que poseen al momento de ingresar a la universidad. La característica del alumnado en la que menos (36,49\%) reparan los docentes es aquella enfocada en los conocimientos de economía al ingresar a la universidad. En consecuencia, la característica del alumnado más destacada por el profesorado es la vocación que tiene el estudiantado por la carrera universitaria que ha elegido, mientras que, en contraparte, los conocimientos generales al ingreso a la universidad y el uso de tutorías por parte del alumnado, son las características menos destacadas por el personal docente.

Tabla 4

Porcentaje y puntuación media del profesorado sobre el nivel que tienen los alumnos respecto a cada característica, Universidad de Cuenca- Ecuador, 2018

Características de los alumnos

Vocación por los estudios seleccionados

Conocimientos generales al ingresar a la universidad

Preparación para el trabajo en equipo a su ingreso en la universidad

Capacidad para el aprendizaje autónomo a su ingreso en la universidad

Conocimientos de economía a su ingreso en la universidad

Conocimientos de matemáticas a su ingreso en la universidad

Actitud hacia el aprendizaje al ingreso en la universidad

Participación activa del alumno en su aprendizaje en el periodo universitario

Participación activa del alumno en las clases presenciales en el periodo universitario

Uso de las tutorías por parte del alumno

Actitud del alumno hacia la innovación docente

Capacidad del alumno para el diseño de su trayectoria formativa

Fuente: elaboración propia, datos recolectados en el marco de proyecto: La evaluación y acreditación en la Educación Superior: el caso de la Universidad de Cuenca, 2017.

\begin{tabular}{cc}
$\%$ & Media \\
\hline 58.68 & 2.35 \\
42.82 & 1.71 \\
48.12 & 1.92 \\
43.24 & 1.73 \\
36.49 & 1.46 \\
44.82 & 1.79 \\
56.14 & 2.25 \\
55.73 & 2.23 \\
57.38 & 2.30 \\
43.68 & 1.75 \\
57.41 & 2.30 \\
49.33 & 1.97
\end{tabular}

Los contenidos de este artículo están bajo una licencia Creative Commons 
Así mismo, fue necesario determinar la apreciación que tienen los docentes sobre las características del alumnado y su asociación con la mejora de la calidad educativa (Tabla 5). Se determinó que, por arriba del 70\%, las características del alumnado referidas a: la actitud hacia el aprendizaje al ingreso en la universidad, la participación activa estudiantil en su aprendizaje en el periodo universitario, y la participación activa del alumnado en las clases, son a las que los docentes atribuyen mayor asociación con la mejora de la calidad, es decir, para los docentes las características del alumnado antes indicadas tienen mayor incidencia en la mejora de la calidad educativa. Otras características del alumnado que son valoradas por el personal docente como elementos que afectan a la mejora de la calidad educativa, entre un $60 \%$ y un $70 \%$ son: la actitud hacia la innovación, la vocación por los estudios seleccionados, la capacidad del alumnado para el diseño de su trayectoria formativa, la capacidad para el aprendizaje autónomo a su ingreso en la universidad, la preparación para el trabajo en equipo a su ingreso en la universidad, los conocimientos generales al ingresar a la universidad, el uso de las tutorías por parte del alumnado, así como los conocimientos de matemáticas al ingreso en la universidad. En el porcentaje más bajo de todos $(54,98 \%)$ el personal docente ve positivamente que la característica de los alumnos que alude a: los conocimientos de economía que tienen al ingresar a la universidad tiene que ver con la mejora de la calidad educativa. Por lo tanto, la opinión predominante es que, el personal docente considera que la característica del alumnado enfocada en la actitud del alumnado hacia el aprendizaje es la que más incide en la mejora calidad educativa, mientras que la característica del alumnado alusiva a los conocimientos sobre economía es la que menos incide, según el personal docente.

Tabla 5

Porcentaje y puntuación media del profesorado sobre el efecto que tienen las características del alumnado en la mejora de la calidad educativa, Universidad de Cuenca- Ecuador, 2018

\begin{tabular}{lcc}
\hline Características del alumnado & P\% & Media \\
\hline Vocación por los estudios seleccionados & 68.18 & 2.73 \\
Conocimientos generales al ingresar a la universidad & 62.88 & 2.52 \\
Preparación para el trabajo en equipo a su ingreso en la universidad & 64.55 & 2.58 \\
Capacidad para el aprendizaje autónomo a su ingreso en la universidad & 66.73 & 2.67 \\
Conocimientos de economía a su ingreso en la universidad & 54.98 & 2.20 \\
Conocimientos de matemáticas a su ingreso en la universidad & 61.90 & 2.48 \\
Actitud hacia el aprendizaje al ingreso en la universidad & 71.30 & 2.85 \\
Participación activa del alumno en su aprendizaje en el periodo universitario & 70.73 & 2.83 \\
Participación activa del alumno en las clases presenciales en el periodo universitario & 70.68 & 2.83 \\
Uso de las tutorías por parte del alumno & 62.00 & 2.48 \\
Actitud del alumno hacia la innovación docente & 69.10 & 2.76 \\
Capacidad del alumno para el diseño de su trayectoria formativa & 67.38 & 2.70 \\
\hline
\end{tabular}

Fuente: elaboración propia, datos recolectados en el marco de proyecto: La evaluación y acreditación en la Educación Superior: el caso de la Universidad de Cuenca, 2018. 
La tabla 6 muestra el coeficiente de correlación biserial por rangos ( $\mathrm{r}_{\mathrm{bRANK}}$ para la relación entre el área en la que enseña el profesorado y las características del alumnado, así como el efecto de éstas en la mejora de la calidad educativa. Acerca de las características del alumnado, se encontraron dos correlaciones muy bajas según las cuales, el personal docente del área técnica, considera que la calidad educativa mejora cuando los estudiantes tienen vocación por la carrera seleccionada, así como cuando disponen de conocimientos de matemáticas a su ingreso en la universidad. Del mismo modo, en cuanto a las características del alumnado y cómo estas se asocian con la calidad educativa, se observó al menos tres correlaciones negativas muy bajas en el área de ciencias médicas y de la salud con las características orientadas a la vocación por los estudios seleccionados, la capacidad para el aprendizaje autónomo al ingresar a la universidad y conocimientos de matemáticas al ingresar a la universidad. En las carreras de las áreas técnicas, se encontraron correlaciones muy bajas, pero significativas, en cuanto a: la preparación de los alumnos para el trabajo en equipo, los conocimientos en economía y los conocimientos en matemáticas al ingresar a la universidad.

Tabla 6

Universidad de Cuenca: correlación de Pearson de las características del alumnado y el perfil académico del profesorado

\begin{tabular}{|c|c|c|c|c|c|c|c|}
\hline \multirow[t]{2}{*}{ Características del alumnado } & & \multicolumn{3}{|c|}{ Nivel del alumnado ${ }^{2}$} & \multicolumn{3}{|c|}{$\begin{array}{l}\text { Efectos sobre la mejora de la } \\
\text { calidad educativa }{ }^{3}\end{array}$} \\
\hline & & 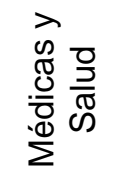 & 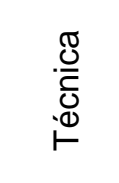 & $\begin{array}{l}\frac{\mathscr{d}}{\frac{\pi}{0}} \\
\frac{0}{0} \\
\dot{0}\end{array}$ & 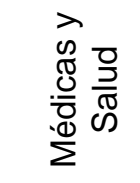 & 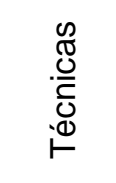 & $\begin{array}{l}\frac{\infty}{\infty} \\
\frac{\omega}{0} \\
\dot{\infty}\end{array}$ \\
\hline $\begin{array}{l}\text { Vocación por los estudios } \\
\text { seleccionados } \\
\text { Conocimientos generales al } \\
\text { ingresar a la universidad } \\
\text { Preparación para el trabajo }\end{array}$ & $\begin{array}{l}\text { Correl. } \\
\text { Sig. bil. } \\
\text { Correl. } \\
\text { Sig. bil. } \\
\text { Correl. }\end{array}$ & $\begin{array}{r}-, 081 \\
, 084 \\
, 017 \\
, 709 \\
-, 010\end{array}$ & $\begin{array}{l}, 135^{\star *} \\
, 004 \\
, 032 \\
, 496 \\
, 038\end{array}$ & $\begin{array}{l}-, 043 \\
, 357 \\
-, 043 \\
, 365 \\
-, 023\end{array}$ & $\begin{array}{c}-, 096^{*} \\
, 045 \\
-, 088 \\
, 067 \\
-, 030\end{array}$ & $\begin{array}{l}, 068 \\
, 156 \\
, 083 \\
, 082 \\
, 111^{*}\end{array}$ & $\begin{array}{l}, 026 \\
, 588 \\
, 006 \\
, 899 \\
-, 069\end{array}$ \\
\hline $\begin{array}{l}\text { en equipo a su ingreso en la } \\
\text { universidad }\end{array}$ & Sig. bil. & ,836 &, 421 & ,620 &, 531 &, 021 &, 154 \\
\hline Capacidad para el & Correl. &,- 039 &, 016 & ,021 &,$- 111^{*}$ & ,077 & ,032 \\
\hline $\begin{array}{l}\text { aprendizaje autónomo a su } \\
\text { ingreso en la universidad }\end{array}$ & Sig. bil. & ,409 & ,744 & ,656 & ,022 & ,110 &, 514 \\
\hline Conocimientos de economía & Correl. &,- 010 & ,065 &,- 046 &,- 090 &, $102^{*}$ &,- 007 \\
\hline $\begin{array}{l}\text { a su ingreso en la } \\
\text { universidad }\end{array}$ & Sig. bil. & ,835 & ,183 & ,342 & ,068 & ,039 & ,881 \\
\hline $\begin{array}{l}\text { Conocimientos de } \\
\text { matemáticas a su ingreso en }\end{array}$ & $\begin{array}{l}\text { Correl. } \\
\text { Sig. bil. }\end{array}$ & $\begin{array}{l}-, 015 \\
, 764 \\
\end{array}$ & $\begin{array}{l}, 106^{*} \\
, 030\end{array}$ & $\begin{array}{l}-, 078 \\
, 113\end{array}$ & $\begin{array}{c}-, 145^{* *} \\
, 003\end{array}$ & $\begin{array}{l}, 219^{* *} \\
, 000\end{array}$ & $\begin{array}{l}-, 061 \\
, 218 \\
\end{array}$ \\
\hline
\end{tabular}

2 Valoración del personal docente sobre el nivel del alumnado respecto a ítem de las características.

${ }^{3}$ Valoración del personal docente sobre el efecto de las características del alumnado en la mejora de la calidad educativa. El término "efecto" no implica relaciones causales en el análisis de datos. 


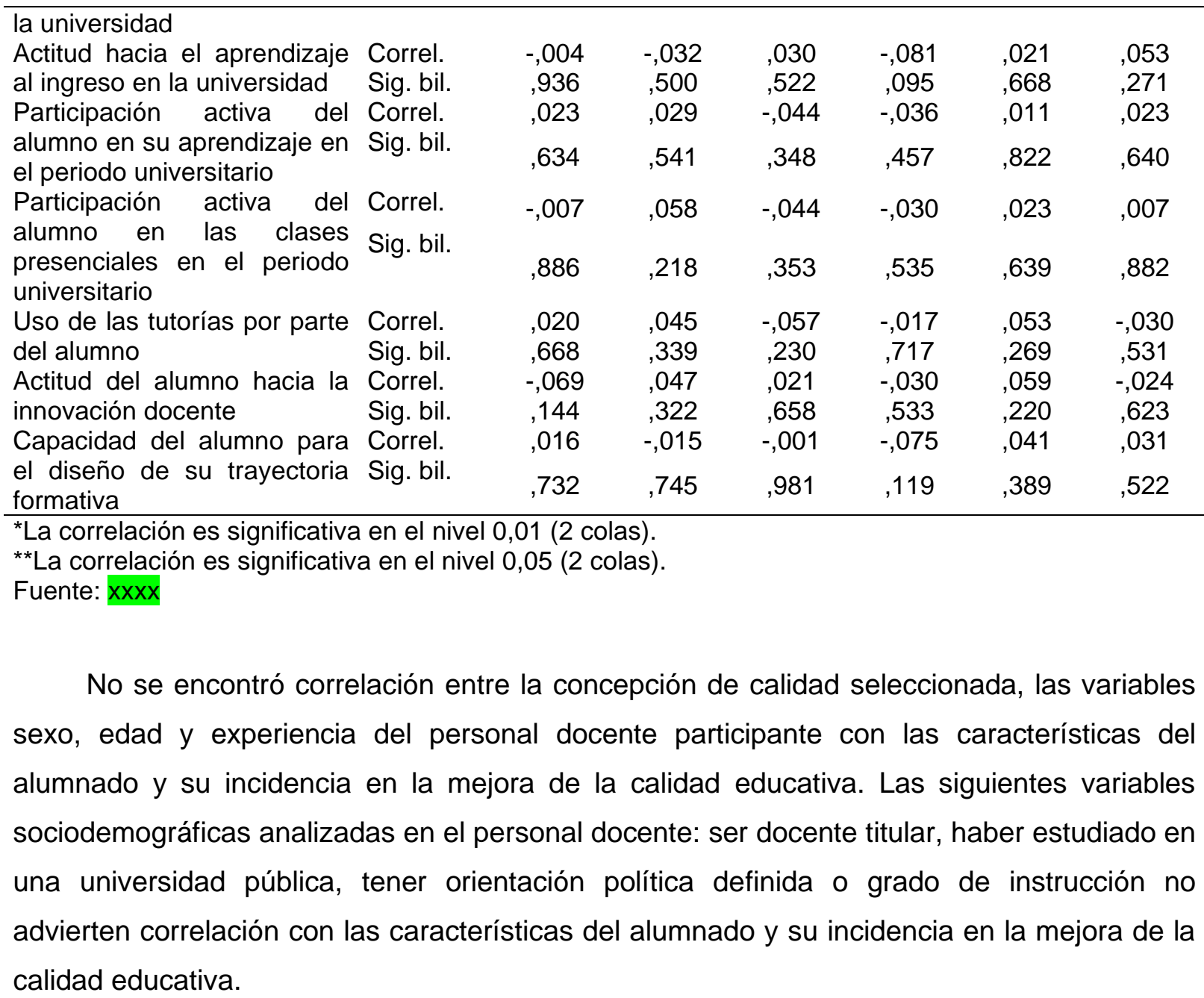

\section{Conclusiones}

El estudio describe la concepción de la calidad educativa universitaria desde la perspectiva del personal docente de la Universidad de Cuenca, Ecuador y la relación con el perfil sociodemográfico y la concepción de calidad educativa del personal docente universitario, por otro lado, identifica las características del alumnado que se consideran importantes para la mejora de la calidad desde la perspectiva docente.

Las concepciones de calidad del personal docente, fue el tema central del presente estudio, de ahí, que uno de los principales resultados evidencie que la mayoría del profesorado afirma apegarse a la concepción de calidad que forma estudiantes capaces de asumir compromiso social, concepción alejada de la medición de excelencia y cumplimiento de estándares con una perspectiva eficientista, que según Boni y Gasper (2011) suele ser la visión imperante en las IES. 
Los resultados permiten concluir que, en la UC, ser docente del área de ciencias sociales está más asociado con la concepción de calidad orientada a la formación de estudiantes capaces de asumir un compromiso social particularmente; concepción de calidad compartida por el colectivo docente que enseña el área médica y de la salud, aunque con una correlación negativa muy baja. De otro lado, el personal docente del área técnica relaciona la calidad con el cumplimiento de estándares. Los resultados evidencian que no existe una concepción única de calidad educativa, de hecho, varía según el área de conocimiento en la cual labora el personal docente; es decir, se puede concluir que las áreas en las cuales enseñan el profesorado determinan la preferencia por una u otra concepción de calidad. Según, Barrenetxea et al. (2015) la rama de enseñanza es una de las variables explicativas más influyente en el concepto de calidad.

Las características sociodemográficas del personal docente no están relacionadas con la concepción de calidad, es decir, ser hombre o mujer, la edad y la experiencia docente no se asocian con la concepción de calidad seleccionada; éste resultado difiere con lo encontrado por Barrenetxea et al. (2015) para quienes las características sociodemográficas y profesionales como el sexo y edad de los docentes si constituyen un determinante al momento de optar por una u otra concepción de calidad.

En el presente estudio, en general, la concepción de calidad con la cual, el personal docente universitario, se siente mayormente identificados es la de formar estudiantes que puedan asumir compromisos sociales. Por otro lado, la concepción individual de cada docente, difiere en la concepción que los docentes creen que tiene la universidad sobre calidad, es decir, para ellos la institución en la que laboran percibe la calidad educativa como el cumplimiento de estándares, en este punto el resultado coincide con la conclusión de Cardona et al. (2009) quienes manifiestan que la concepción de calidad de la IES visualiza una concepción de calidad distinta a la que tienen los catedráticos individualmente.

A la luz de los hallazgos del estudio, podemos concluir que la concepción de calidad del personal docente se inclina por una visión más apegada al desarrollo humano centrada en la formación de sus estudiantes con responsabilidad social, ello no necesariamente implica que la visión individual coincida con la concepción de calidad que se percibe a nivel institucional, tal como se puede apreciar; el personal docente encuestado considera que la UC adopta una concepción de calidad ajena a la suya propia. Esta discrepancia podría ser considerada para analizar, en futuras investigaciones, las ventajas y desventajas de las distintas concepciones y cómo ello determina los procesos de evaluación y acreditación. 
La variación de la concepción de calidad en cada área del conocimiento ratifica el carácter polisémico del término; el término está cruzado por una serie de elementos que no necesariamente subyacen a los ámbitos disciplinares; la variedad de concepciones entre las áreas de conocimiento también evidencia lo que la literatura ha recogido ampliamente en referencia a que la concepción de calidad varía en función de los stakeholders y de la visión que cada uno de ellos tiene sobre la finalidad de las IES.

Los resultados evidencian además, que el personal docente destaca las siguientes características del estudiantado como elementos que afectan a la mejora de la calidad educativa: la vocación por los estudios seleccionados, actitud frente a la innovación docente y participación activa coincidiendo con los hallazgos encontrados en el estudio de Cardona et al. (2009); de igual manera, con el estudio de Garbanzo (2007) quien sostiene que la elección del área de estudio, según los intereses del estudiante, es determinante para el rendimiento y la calidad educativa.

Las características del alumnado, constituyen un elemento relevante que el profesorado consideran afecta la mejora de la calidad educativa, aunque el análisis no se ha realizado en función de causa efecto, se considera que la característica del alumnado referida a la vocación por los estudios seleccionados es la que más efecto tiene en la mejora de calidad educativa de la UC. Podría pensarse que estos estudiantes son más proactivos en los procesos de aprendizaje y, si bien ese no es el parámetro más importante al momento de medir la calidad, si constituye un elemento relevante para el análisis de la calidad y su mejora. Finalmente, en concordancia a lo anterior, la presente investigación deja abierta la posibilidad de plantear otros estudios que indaguen la visión de los estudiantes sobre la calidad educativa, los procesos de evaluación de la calidad y los procesos de acreditación desde la perspectiva del alumnado y otros actores involucrados en educación superior.

\section{Agradecimientos}

Agradecemos especialmente al Departamento de Investigación de la Universidad de Cuenca (DIUC) por el apoyo y financiamiento al proyecto denominado: La evaluación y acreditación en la Educación Superior: el caso de la Universidad de Cuenca, proyecto ganador del XV concurso universitario de investigación del cual se desprende el presente estudio. 


\section{Referencias}

Aguila, Vistremundo. (2005). El concepto calidad en la educación universitaria: clave para el logro de la competitividad institucional. Revista iberoamericana de Educación, 36(12), 1-7.

Alvarado-Lagunas, Elías., Luyando-Cuevas, José-Raúl., y Picazzo-Palencia, Esteban. (2015). Percepción de los estudiantes sobre la calidad de las universidades privadas en Monterrey. Revista iberoamericana de educación superior, 6(17), 58-76. Doi https://dx.doi.org/10.1016/i.rides.2015.10.003

Araujo, Sonia. y Trotta, Lucía. (2011). La acreditación de las Ingenierías: configuración compleja en la institucionalización de la política. Archivos de Ciencias de la Educación, 5(5), 83-97.

Araya Leticia., Arias Karina. e Ibarra Américo. (2009). Sistema de Educación Superior en Chile. En Jon Olaskoaga (coord.), Hacia una educación superior de calidad, un análisis desde la perspectiva del profesorado en Argentina, Chile, España y México (pp. 39-59). La Plata, Argentina: Universidad Nacional de la Plata.

Barrenetxea, Miren; Cardona, Antonio, Mijangos, Juan José y Olaskoaga Jon. (2009) Sistema universitario español. En Jon Olaskoaga (coord.), Hacia una educación superior de calidad, un análisis desde la perspectiva del profesorado en Argentina, Chile, España y México (pp. 61-80). La Plata, Argentina: Universidad Nacional de la Plata.

Barrenetxea Miren; Olaskoaga Jon, González Xabier, Barandiaran Marta, Cardona Antonio, Mijangos Juan José y Onaindia Eneritz. (2015). Nociones de calidad de los académicos españoles y determinantes socio-profesionales de las mismas. Procedia-Social and Behavioral Sciences, 196, 8-13. Doi: https://doi.org/10.1016/i.sbspro.2015.07.002

Barandiaran Marta. (2013). El concepto de calidad en la educación superior, una estrategia de apoyo desde las universidades a la generación de desarrollo humano (Tesis doctoral). Universidad del País Vasco, España. Disponible en http://www.hegoa.ehu.es/es/articles/text/nueva tesis doctoral en hegoa33333

Becher, Tony (1999) Quality in the professions, Studies in Higher Education, 24(2), 225-235. DOI: $10.1080 / 03075079912331379908$

Boni Alexandra y Gasper Des (2011). La Universidad como debiera ser. Propuestas desde el desarrollo humano para repensar la calidad de la Universidad. Sistema. Revista de ciencias sociales, (220), 99-115.

Cardona, Antonio., Barrenetxea, Miren., Mijangos, Juan José., Olaskoaga, Jon. (2009). Concepto y Determinantes de la Calidad de la Educación Superior. Un Sondeo de Opinión entre Profesores de Universidades Españolas. Archivos Analíticos de Políticas Educativas, 17(10), 1-25. 
Crozier, Fiona., Curvale, Bruno., Dearlove, Rachel., Hell, Emmi. y Hénard, Fabrice. (2006). Terminology of quality assurance: towards shared European values? ENQA Occasional Papers 12. Recuperado de http://www.enqa.eu/pubs.lasso

Garbanzo, Guiselle. (2007). Factores asociados al rendimiento académico en estudiantes universitarios, una reflexión desde la calidad de la educación superior pública. Educación, 31(1), 43-63.

Harvey, Lee. and Green, Diana. (1993). Defining Quality, Assessment \& Evaluation in Higher Education, 18:1, 9-34. Doi: http://dx.doi.org/10.1080/0260293930180102

Harvey, Lee and Williams, James. (2010). Fifteen Years of Quality in Higher Education (Part Two), Quality in Higher Education, 16(2), 81-113, DOI: 10.1080/13538322.2010.485722

Hughey, Aaron. (1997). What higher education can learn from business and industry? Industry and Higher Education, 11(2), 73-78.

Leite, Denise., y Genro, Maria Elly Herz. (2012). Avaliação e internacionalização da educação superior: Quo vadis América Latina? Avaliação: Revista da Avaliação da Educação Superior (Campinas), 17(3), 763-785. Doi https://doi.org/10.1590/S1414$\underline{40772012000300009}$

Ley Orgánica Reformatoria a Ley Orgánica De Educación Superior. (2018). Registro Oficial № 297. Recuperado de: http://aka-cdn.uce.edu.ec/ares/tmp/Elecciones/2\%20LOES.pdf

López-Segrera, Francisco. (2012). La Segunda Conferencia Mundial de Educación Superior (UNESCO, 2009) y la visión del concepto de acreditación en las conferencias de UNESCO (1998-2009). Avaliação: Revista da Avaliação da Educação Superior, 17(3), 619-636.

Martínez, Rosario., Castellanos, Miguel Ángel., y Chacón, José Carlos. (2015). Análisis de Datos en Psicología y Ciencias de la Salud (Volumen II: Inferencia Estadística). Madrid: EOS.

Marúm, Elia., Cortés, José., Padilla, Ruth., Curiel, Federico. y Robles, Ma. Lucila. (2009). Sistema universitario mexicano. En Jon Olaskoaga (coord.), Hacia una educación superior de calidad, un análisis desde la perspectiva del profesorado en Argentina, Chile, España y México (pp. 83-105). La Plata, Argentina: Universidad Nacional de la Plata.

Mateo, Joan. (2009). La investigación ex post - facto. En Rafael Bisquerra, Metodología de la investigación educativa (pp. 187-221). Madrid, España: Arco/ Libros - La Muralla.

McMillan James. y Schumacher Sally. (2005). Investigación educativa una introducción conceptual. Pearson.

Olaskoaga, Jon (coord.). (2009). Hacia una educación superior de calidad. Un análisis desde la perspectiva del profesorado en Argentina, Chile, España y México. La Plata, Argentina: Universidad Nacional de la Plata. 
Olaskoaga, Jon., Marúm, Elia. y Partida, Inés. (2015). La diversidad semántica y el carácter político de las nociones de calidad en la Educación Superior de México. Revista de La Educación Superior, $\quad$ 44(173), $\quad$ 85-102. Doi https://doi.org/https://doi.org/10.1016/..resu.2015.04.003

Palmer, Alfonso., Jiménez, Rafael., y Montaño, Juan José. (2000). Tutorial sobre coeficientes de correlación con una o dos variables categóricas. Revista Electrónica de Psicología, $4(2)$.

Persoglia Laura, López Martín y Zoloa Juan (2009). Percepción del profesorado universitario sobre los factores ligados a la calidad de la enseñanza: resultados de la Argentina. En Jon Olaskoaga (coord.), Hacia una educación superior de calidad, un análisis desde la perspectiva del profesorado en Argentina, Chile, España y México (pp. 83-105). La Plata, Argentina: Universidad Nacional de la Plata.

Prisacariu, Anca. y Shah, Mahsood. (2016). Defining the quality of higher education around ethics and moral values. Quality in Higher Education, 22(2), 152-166, DOI: 10.1080/13538322.2016.1201931

Ramirez, René. (2013). Tercera ola de transformación de la educación superior en Ecuador. Quito. Recuperado de http://www.educacionsuperior.gob.ec/wpcontent/uploads/downloads/2013/08/Tercera-ola-de-transformaci\%C3\%B3n-de-laeducaci\%C3\%B3n-superior-en-Ecuador3.Pdf

Rengifo-Millán, Maritza. (2017). Calidad en la Educación Superior desde las Ciencias Sociales y Administrativas. Revista Latinoamericana de Ciencias Sociales, Niñez y Juventud, 15(2), 1251-1270. DOI: http://dx.doi.org/10.11600/1692715x.1523113092016

Roig, Arturo. (2014). El modelo de evaluación de las universidades ecuatorianas. Apuntes críticos para el debate. Recuperado de https://www.eluniverso.com/sites/default/files/archivos/2014/04/manifiesto el modelo d e evaluacion de las universidades ecuatorianas.pdf

Rovelli, Laura. (2018). Las Conferencias mundiales y Regionales de Educación Superior como instrumentos de política. Mediaciones y reformulaciones latinoamericanas de fines del siglo XX y principios del XXI. En Claudio Suasnábar, Damián Del Valle (coord.), Política y tendencias de la educación superior a diez años de la CRES 2008 (pp. 57-72). Ciudad Autónoma de Buenos Aires: IEC - CONADU: CLACSO.

Van Kemenade, Everard., Pupius, Mike. y Hardjono, Teun. (2008). More Value to Defining Quality, Quality in Higher education, 14(2), 175-185, DOI: $\underline{10.1080 / 13538320802278461}$

Villavicencio, Arturo. (2014). El modelo de evaluación de carreras: más de lo mismo. Boletín informativo Spondylus. Universidad Andina Simón Bolívar. Quito. Recuperado de: http://www.uasb.edu.ec/UserFiles/372/File/pdfs/PAPER\%20UNIVERSITARIO/2015/Mo delo Evaluacion Carreras.Pdf 
Revista indizada en

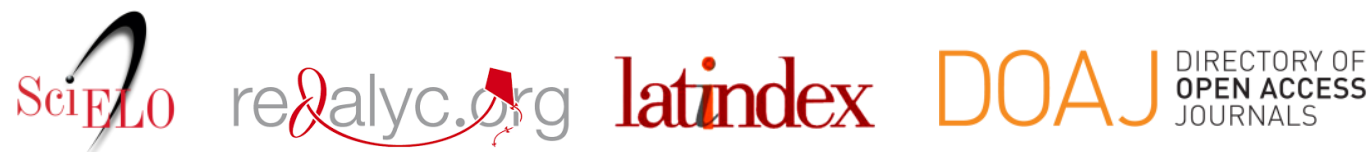

Distribuida en las bases de datos:

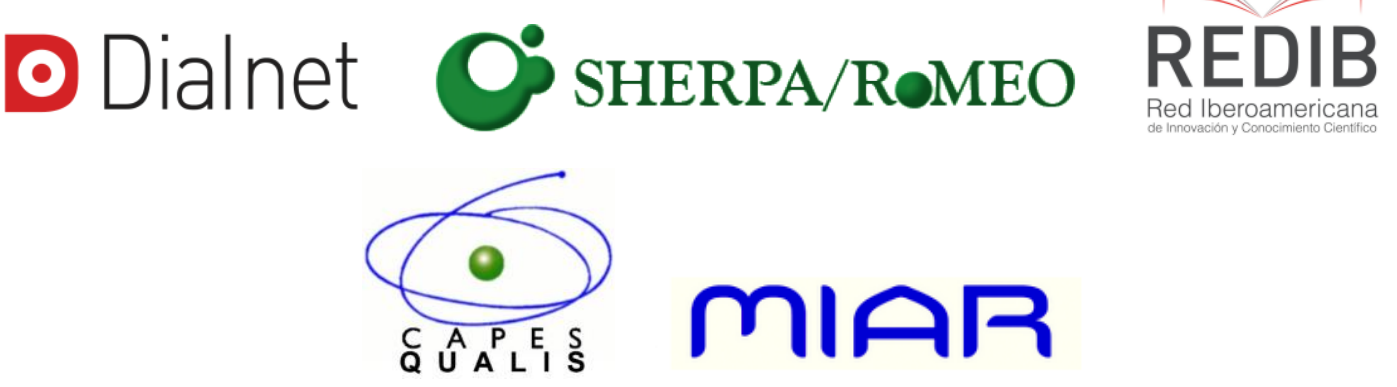

